\title{
15-oxoeicosatetraenoic acid mediates monocyte adhesion to endothelial cell
}

\author{
Guohua Ma ${ }^{1,3+}$, Bing Pan ${ }^{2 \dagger}$, Sufen Ren ${ }^{1}$, Caixia Guo ${ }^{1}$, Yansong Guo ${ }^{4}$, Lixin Wei ${ }^{5}$, Lemin Zheng ${ }^{2 *}$ and \\ Buxing Chen $^{1^{*}}$ (1)
}

\begin{abstract}
Background: A great number of studies reported that 12/15-lipoxygenase (12/15-LO) played an important role in atherosclerosis. And its arachidonic acid(AA) metabolite, 15(S)-hydroperoxy-5,8,11,13-(Z,Z,Z,E)-eicosatetraenoic acid (15(S)-HETE), is demonstrated to mediate endothelial dysfunction. 15-oxo-5,8,11,13-(Z,Z,Z,E)-eicosatetraenoic acid (15-OxO-ETE) was formed from 15-hydroxyprostaglandin dehydrogenase (PGDH)-mediated oxidation of 15(S)-HETE. However, relatively little is known about the biological effects of 15-oxo-ETE in cardiovascular disease. Here, we explore the likely role of 15-lipoxygenase (LO)-1-mediated AA metabolism,15-oxo-ETE, in the early pathogenesis of atherosclerosis.
\end{abstract}

Methods: The 15-oxo-ETE level in serum was detected by means of liquid chromatography and online tandem mass spectrometry (LC-MS/MS). And the underlying mechanisms were illuminated by molecular techniques, including immunoblotting, MTT assay, immunocytochemistry and Immunohistochemistry.

Results: Increased 15-oxo-ETE level is found in in patients with acute myocardial infarction (AMI). After 15-oxo-ETE treatment, Human umbilical vein endothelial cells (HUVECs) showed more attractive to monocytes, whereas monocyte adhesion is suppressed when treated with PKC inhibitor. In ex vivo study, exposure of arteries from C57 mice and ApoE-/-mice to 15-oxo-ETE led to significantly increased E-selectin expression and monocyte adhesion.

Conclusions: This is the first report that 15-OxO-ETE promotes early pathological process of atherosclerosis by accelerating E-selectin expression and monocyte adhesion. 15-OxO-ETE -induced monocyte adhesion is partly attributable to activation of PKC.

Keywords: 15-oxo-ETE, monocyte adhesion, E-selectin, Atherosclerosis, PKC

\section{Background}

Arachidonic acid (AA) is a key polyunsaturated fatty acid (PUFA), which derives a great number of bioactive mediators that are involved in many physiological and pathological processes. AA can be oxygenated by a variety of different enzymes, including lipoxygenases, cyclooxygenases, and cytochrome P450s [1], this study will focus on the biosynthesis of 15-oxo-5,8,11,13(Z,Z,Z,E)-eicosatetraenoic acid (15-oxo-ETE) in 15-LOX

\footnotetext{
* Correspondence: zhengl@bjmu.edu.cn; chbux@126.com

${ }^{\dagger}$ Equal contributors

${ }^{2}$ The Institute of Cardiovascular Sciences and Institute of Systems Biomedicine, School of Basic Medical Sciences, and Key Laboratory of Molecular Cardiovascular Sciences of Ministry of Education, Peking University Health Science Center, Beijing 100191, China

'Department of Cardiology, Beijing Tian Tan Hospital, Capital Medical University, Beijing 100050, China

Full list of author information is available at the end of the article
}

pathway. Two types of 15-LOX have been identified in human, including 15-LOX-1 (encoded by ALOX15 gene) and 15-LOX-2(encoded by ALOX15B gene) [2, 3]. In humans, 15-LOX is expressed in various tissue cells and plays different physiopathologic function [2, 4]. Notably, 15-LOX is highly expressed in macrophages within human atherosclerotic plaques [5-7]. In macrophages, 15LOX-1 converts AA to 15-hydroperoxyeicosatetraenoic acids (15-HpETEs), which is soon reduced by peroxidase to 15-hydroxyeicosatetraenoic acids (15-HETEs). Finally, 15 -HETEs was oxidized to 15-oxoETE by 15 hydroxyprostaglandin dehydrogenase (15-PGDH), which requires $\mathrm{NAD}+$ as a cofactor.

There is substantial evidence for the pro-atherosclerotic effect of 15-LOX: (i) downregulation of 12/15-LOX reduced lipid oxidation and foam cell formation [8]; (ii) 
up-regulation of 15-LOX-2 in macrophages enhances inflammation and the recruitment of inflammatory cells in hypoxic atherosclerotic plaque [6]; (iii) 15-LOX is a key enzyme for the oxidization of low-density lipoprotein (LDL) [9-11]; (iv) disruption of 12/15-LOX gene decreased high-fat diet induced endothelial dysfunction and vascular inflammation [12]; (v) ALOX15B is highly expressed in carotid lesions in patients with cerebrovascular symptoms. 15-HETE, as the initial 15-LOX metabolite of AA, was first identified in human atherosclerotic plaque using high-performance liquid chromatography [13]. It is reported that 15S-HETE may be involved in endothelial dysfunction, monocyte/macrophage transmigration [12], vascular wall remodeling [14], and angiogenesis [15]. Moreover, a recent research showed that 15-HETE promoted oxidative stress and foam cell formation [16]. However, the evidence for the role of 15-oxoETE in atherosclerosis is unclear.

A large body of evidence suggests that endothelial dysfunction is a key variable in the pathogenesis of atherosclerosis [17-19]. Endothelial dysfunction, as a comprehensive index of the overall CVD risk factor burden includes three main consequences, exposure of adhesion molecules, the activation and aggregation of platelets, cholesterol accumulation [20]. Monocyte recruitment is the initial incident in atherosclerotic plaque formation, the present study was designed to explore the likely effect of 15-oxo-ETE on monocyte recruitment.

\section{Methods}

\section{Study population}

We enrolled patients with diagnosis of acute myocardial infarction (AMI) $(n=8)$ and patients without coronary artery lesion admitted for elective coronary diagnostic $(n=8)$. In addition, all the subjects didn't take aspirin or other NSAIDs until first medical contact in Beijing TianTan hospital.

\section{Measurement of AA metabolites}

Blood samples for 15-oxoETE assays were drawn into EDTA tubes from a radial artery approach before coronary angiography. After centrifugation, blood samples were stored at $-80{ }^{\circ} \mathrm{C}$ until AA derivative measurement. Plasma AA derivative levels were quantified by ultra-high performance liquid chromatography-tandem mass spectrometric (UPLC-MS/MS). Briefly, Waters Oasis-HLB cartridges were washed with $1 \mathrm{ml}$ methanol and $1 \mathrm{ml} \mathrm{MilliQ}$ water, and samples with internal standard mixture (each $5 \mathrm{ng}$ ) were loaded into the cartridges and washed with $5 \%$ methanol $(1 \mathrm{ml})$. The aqueous plug was then pulled from the SPE cartridges under high vacuum for $20 \mathrm{~min}$, and the analytes were eluted into tubes with $1 \mathrm{ml}$ methanol. Samples were dried using a Thermo Speed Vac, dissolved in $120 \mu \mathrm{l} 30 \% \mathrm{ACN}$, and filtered with a $0.22 \mu \mathrm{C}$ for UPLC-MS/MS. A total of 31 AA mediators were detected.

\section{5-oxoETE and animals}

15-oxoETE was purchased from Cayman Chemical (Ann Arbor, MI). C57 mice (6-8-week-old males) and ApoE-/mice (6-8-week-old males) were obtained from the animal house of Peking University, Beijing, China.

\section{Cell lines and cell culture}

The human monocyte cell line THP-1 was purchased from Cell Resource Center, Institute of Basic Medical Sciences, Chinese Academy of Medical Sciences, Beijing, China. Cells were cultured in RPMI1640medium (Hyclone, USA) containing 10\% fetal bovine serum. HUVECs were isolated from umbilical veins [21]. The cells were cultured in ECM (ScienCell, USA) containing 5\% FBS, $1 \%$ endothelial cell growth supplement, and $1 \%$ penicillin/streptomycin solution. The cells were then cultured in a humidified incubator of $5 \% \mathrm{CO}_{2}$ at $37{ }^{\circ} \mathrm{C}$. HUVECs were used at passages 2-5.

\section{3-(4,5-Dimethylthiazol-2-yl)-2,5-diphenyltetrazolium bromide (MTT) assay}

For cell viability assay, HUVECs $\left(5 \times 10^{3}\right.$ cells/well $)$ were seeded in 96-well plates and cultured overnight, subsequently treated with varying concentrations of 15-oxoETE $(0,2.5,5,10,20,40 \mu \mathrm{mol} / \mathrm{L})$ for $24 \mathrm{~h}$ or 15-oxoETE $(0,0.5,1,2,4,8 \mu \mathrm{mol} / \mathrm{L})$ for $12 \mathrm{~h}$. And then added $10 \mu \mathrm{l}$ MTT solution to each well at a final concentration of $0.5 \mathrm{mg} / \mathrm{ml}$ and incubated for an additional $4 \mathrm{~h}$. At the end of incubation, dimethylsulfoxide $(150 \mu \mathrm{l})$ was added to each well and then the absorbance was measured at $570 \mathrm{~nm}$ using an enzyme-linked immunosorbent assay (ELISA) reader.

\section{Measurement of adhesion molecules expression in HUVECs}

Adhesion molecules expression was measured by immunocytochemistry. Briefly, HUVECs were plated in 96-well plates. Confluent HUVECs were serum starved for $6 \mathrm{~h}$ and treated with 15-oxoETE $(0,0.5,1,2,4$, $8 \mu \mathrm{mol} / \mathrm{L}$ ) for $6 \mathrm{~h}$ at $37^{\circ} \mathrm{C}$, TNF- $\alpha$ was served as positive control. Then HUVECs were incubated with Rabbit Anti-ICAM-1 (PB0053, Boster) or Rabbit Anti -VCAM1 (BA0406, Boster) or Rabbit Anti -E-selectin (BA0615, Boster) (1:200; Boster, China) in First Antibody Dilution Buffer for $2 \mathrm{~h} 37^{\circ} \mathrm{C}$. Omission of primary antibody was conducted in Negative controls. And then cells were incubated with a horseradish peroxidase-conjugated antibody (1:1000, Abcam, USA) for $1.5 \mathrm{~h}$ at $37{ }^{\circ} \mathrm{C}$. Quantification was performed by measuring the absorbance at $450 \mathrm{~nm}$ by a TMB peroxidase EIA substrate kit (Bio-Med Innovation, China) [22]. 


\section{In vitro adhesion assay}

In brief, confluent HUVECs on 96-well plates were starved for $6 \mathrm{~h}$ and treated with 15-oxoETE $(0,0.5,1,2$, $4,8 \mu \mathrm{mol} / \mathrm{L})$ for $6 \mathrm{~h}$ at $37{ }^{\circ} \mathrm{C}$. Thereafter, cells were exposed to human THP-1 monocytes $\left(10^{5}\right.$ cells/well) for 40 min. Non-adherent THP-1 cells were removed by washing one time with PBS, the absorbance of adherent cells at $450 \mathrm{~nm}$ was measured. Six parallel wells were set up for each group. Adherent cells were counted in randomly selected optical fields taken from an inverted microscope (Leica). PKC inhibitor, staurosporine $(2.5 \mathrm{nmol} /$ $\mathrm{L})$, was used at the beginning of treatment [23].

\section{PKC activity assay}

The PKC activity in the HUVEC lysates was determined by PepTag ${ }^{\bullet}$ Non-Radioactive Protein Kinase Assays (Promega, V5330, USA). As described in the manufacturer's instructions, $5 \times 10^{6}$ cells were removed to prepare PKC extracts. The brightly colored, fluorescent peptide substrate was specially phosphorylated by PKC, and its net charge was altered from +1 to -1 . This change in the net charge of the substrate allowed the phosphorylated and non-phosphorylated versions of the substrate to be rapidly separated on an agarose gel [22].

\section{Immunoblotting}

Bicinchoninic acidprotein assay kit was purchased from Thermo Fisher Scientific (USA). E-selectin (ab18981) and secondary antibodies was purchased from Cell Signaling Technology (USA). Details of immunoblotting have been described elsewhere [24].

\section{Immunohistochemistry}

Histology tissues were fixed in 4\% paraformaldehyde and subsequently embedded in paraffin wax. $6-\mu \mathrm{m}$-thick sections were cut from each paraffin-embedded tissue and collected on microscope slides, and then dewaxed with xylene and rehydrated with $\mathrm{dd}_{2} \mathrm{O}$. Endogenous peroxidases were blocked by $0.3 \% \mathrm{H}_{2} \mathrm{O}_{2}$ solution (dilute with methanol) for $12 \mathrm{~min}$. Antigen retrieval was conducted in EDTA working solution (10 $\mathrm{mmol} / \mathrm{L}, \mathrm{pH} 8.0)$ for $10 \mathrm{~min}$ at $92{ }^{\circ} \mathrm{C}$. The tissue sections were blocked by $5 \%$ goat serum for $1.5 \mathrm{~h}$ at $37{ }^{\circ} \mathrm{C}$, after which sections were incubated with primary antibodies (rabbit anti-ICAM-1 (PB0054, Boster), VCAM-1 (BA0406, Boster), or Eselectin (BA0615, Boster); dilution 1:200) overnight at $4{ }^{\circ} \mathrm{C}$. The tissue sections were then treated with appropriate HRP-conjugated secondary antibodies for $40 \mathrm{~min}$ at $37{ }^{\circ} \mathrm{C}$, and Antigen-antibody reactions were stained with 3, 3-diaminobenzidine. The adhesion molecules expression was examined with a Nikon Eclipse $\mathrm{Ti}$ microscope under high power $(400 \times)$ fields. Adhesion molecules expression was quantified by Leica Q550CW image analysis system.

\section{Ex vivo adhesion assay}

For ex vivo adhesion experiments, $150 \mu \mathrm{l}$ PBS and $150 \mu \mathrm{l} 15$-oxoETE $(314 \mu \mathrm{M})$ were respectively given by tail vein injection. $24 \mathrm{~h}$ later, mice were anaesthetized with ketamine and 5\% chloral hydrate. Under sterile conditions, the heart was exposed through a left thoracotomy, and perfused from the left ventricle with normal saline until the blood was washed out. Then Hoechstlabeled THP-1 monocytes $\left(1 \times 10^{7}\right.$ cells $\left./ \mathrm{ml}\right)$ were perfused from the left ventricle to the aorta and incubated for $45 \mathrm{~min}$ at $37{ }^{\circ} \mathrm{C}$. Non-adherent THP-1 cells were removed by perfusion with normal saline. Aortas were harvested at the level of the aortic arch to the abdominal aorta beyond renal arteries and subsequently fixed in $4 \%$ paraformaldehyde solution. Adhesion was observed by confocal laser scanning microscope and quantified by calculating the areas of fluorescent monocytes attached to the vascular surface.

\section{Statistical analysis}

Statistical analysis was carried out using one way ANOVA with Dunnett's post-test and Student $t$ test; $p<0.05$ was considered significant. Error bars represent mean $\pm \mathrm{SD}$.

\section{Result}

\section{Patient clinical characteristics}

A total of 16 patients were included in the study. Among enrolled patients, 8 patients presented with stable angina pectoris, 4 patients with non ST-segment elevated MI and 4 patients with ST-elevated MI. The coronaries of patients in control group are all normal or close to normal. Patients in AMI group all have one an infarctedrelated artery with 80\%-100 stenosis. Patient clinical characteristics are summarized in Table 1. The details of patients are provided in Additional file 1.

Table 1 The clinical characteristics of patients are summarized as follow

\begin{tabular}{lll}
\hline Variable & Control & AMl \\
\hline Age (years) (mean \pm SD) & $56.4 \pm 8.5$ & $60.0 \pm 8.8$ \\
Male sex (male/total) & $7(87.5)$ & $7(87.5)$ \\
Hyperlipidemia (n, \%) & $7(87.5)$ & $7(87.5)$ \\
Diabetes mellitus (n) & 0 & 0 \\
Hypertension (n, \%) & $4(50)$ & $6(75)$ \\
Smoking (n, \%) & $7(87.5)$ & $7(87.5)$ \\
History of ischemic heart disease (n) & 0 & 0 \\
Clinical presentation & & \\
$\quad$ SAP(n, \%) & $8(100)$ & $0(0)$ \\
$\quad$ Non-STEMI(n, \%) & $0(0)$ & $4(50)$ \\
STEMI(n, \%) & $0(0)$ & $4(50)$ \\
\hline
\end{tabular}




\section{AA metabolites of two groups}

We found that several AA metabolite components showed difference between control and AMI groups (Table 2). Among CYP450 pathway metabolites, 11,12DHET $(P<0.05)$, 14,15-DHET $(P<0.01)$, 16-HETE $(P<0.05)$, 18-HETE $(P<0.01)$, 20-HETE $(P<0.01)$, 5,6DHET $(P<0.01)$ and $8,9-$ DHET $(P<0.05)$ levels were significant higher in AMI group compared with control group. However, no difference is found in COX_6 kPGF1a while other metabolites in COX pathway were undetectable. Interestingly, among 15-LOX pathway, 15-HETE and 15-oxoETE are both higher in AMI

Table 2 A total of 31 AA mediators were detected by UPLC-MS/ MS, "-" means that the compound was measured but was undetectable

\begin{tabular}{|c|c|c|c|c|}
\hline & & Control $(n=8)$ & AMI $(n=8)$ & $P$ \\
\hline & $A A^{*}$ & $104,946 \pm 50,057$ & $199,020 \pm 104,954$ & 0.0382 \\
\hline \multirow[t]{8}{*}{$\operatorname{cox}$} & 6 k-PGF1a & $3.4780 \pm 1.8930$ & $3.3490 \pm 2.1540$ & 0.9008 \\
\hline & 15d-PGJ2 & - & - & \\
\hline & PGB2 & - & - & \\
\hline & PGD2 & - & - & \\
\hline & PGE2 & - & - & \\
\hline & PGF2a & - & - & \\
\hline & PGJ2 & - & - & \\
\hline & TXB2 & - & - & \\
\hline \multirow[t]{9}{*}{ LOX } & 11-HETE* & $0.4650 \pm 0.3027$ & $1.020 \pm 0.5969$ & 0.0343 \\
\hline & 12-HETE & $4.2260 \pm 2.5060$ & $11.2100 \pm 10.5400$ & 0.0896 \\
\hline & 15-HETE* & $1.5640 \pm 0.7041$ & $4.0450 \pm 2.368$ & 0.0131 \\
\hline & 15-OXO-ETE* & $0.0650 \pm 0.0424$ & $0.1788 \pm 0.1391$ & 0.0441 \\
\hline & 5-HETE* & $0.8913 \pm 0.4127$ & $1.6000 \pm 0.0672$ & 0.0233 \\
\hline & 5-OXo-ETE & $0.9000 \pm 0.0298$ & $0.1525 \pm 0.159$ & 0.2944 \\
\hline & 8-HETE** & $0.4438 \pm 0.2850$ & $0.9000 \pm 0.3100$ & 0.0084 \\
\hline & 9-HETE* & $0.3688 \pm 0.2658$ & $0.8425 \pm 0.4803$ & 0.0285 \\
\hline & LTB4 & $0.0243 \pm 0.0229$ & $0.0425 \pm 0.0385$ & 0.2950 \\
\hline \multirow[t]{13}{*}{ CYP450 } & 11,12-DHET* & $0.1888 \pm 0.0564$ & $0.2975 \pm 0.0932$ & 0.0135 \\
\hline & 11,12-EET & $0.1988 \pm 0.1133$ & $0.2400 \pm 0.0919$ & 0.4374 \\
\hline & 14,15-DHET** & $0.4013 \pm 0.1015$ & $0.6450 \pm 0.1706$ & 0.0037 \\
\hline & 14,15-EET & $0.2188 \pm 0.1228$ & $0.3425 \pm 0.1276$ & 0.0681 \\
\hline & 17-HETE & - & - & \\
\hline & 19-HETE & - & - & \\
\hline & 16-HETE* & $0.0750 \pm 0.0093$ & $0.1386 \pm 0.0684$ & 0.0214 \\
\hline & 18-HETE** & $0.1250 \pm 0.0367$ & $0.2338 \pm 0.0902$ & 0.0070 \\
\hline & 20-HETE** & $0.5312 \pm 0.1997$ & $1.3840 \pm 0.6527$ & 0.0031 \\
\hline & $5,6-\mathrm{DHET}^{* *}$ & $0.0875 \pm 0.0315$ & $0.1850 \pm 0.0731$ & 0.0038 \\
\hline & 5,6-EET & $0.6525 \pm 0.4443$ & $0.7238 \pm 0.4517$ & 0.7551 \\
\hline & 8,9-DHET* & $0.1000 \pm 0.0293$ & $0.1438 \pm 0.0403$ & 0.0263 \\
\hline & 8,9-EET & $0.1700 \pm 0.0894$ & $0.2325 \pm 0.0836$ & 0.1708 \\
\hline
\end{tabular}

${ }^{*} P<0.05,{ }^{* *} P<0.01$ (unit:ng $/ \mathrm{ml}$ ) group than control group $(P<0.05)$ (Fig. 1), indicating their likely pro-atherosclerosis role in AMI.

Cell viability of HUVECs undergoing 15-oxoETE treatment To explore the influence of 15-oxoETE on cell viability, MTT assay were conducted on HUVECs treated with varying doses $(0-40 \mu \mathrm{mol} / \mathrm{L})$ of 15 -oxoETE for a $24 \mathrm{~h}$ period. The cells exhibited a dose-dependent reduction in viability (Fig. 2a). Endogenous 15-oxoETE is so low that it can't kill cells in short period. Therefore, lower doses of 15-oxoETE $(0-8 \mu \mathrm{mol} / \mathrm{L})$ were added to cultured HUVECs under serum deprivation, and then the cell viability was assessed by MTT assay after $12 \mathrm{~h}$. HUVECs showed no change when treated with concentrations of $0-8 \mu \mathrm{mol} / \mathrm{L}$, which will be applied to the subsequent experiments (Fig. 2b).

\section{5-oxo-ETE promotes monocyte adhesion and positively regulates E-selectin expression}

Monocyte adhesion is a vital process in the pathogenesis of atherosclerosis. To uncover the effect of 15-oxo-ETE on monocyte adhesion to HUVECs, HUVECs were treated with various doses of 15-oxo-ETE for $6 \mathrm{~h}$ and co-cultured with THP-1 cells for an additional $40 \mathrm{~min}$.

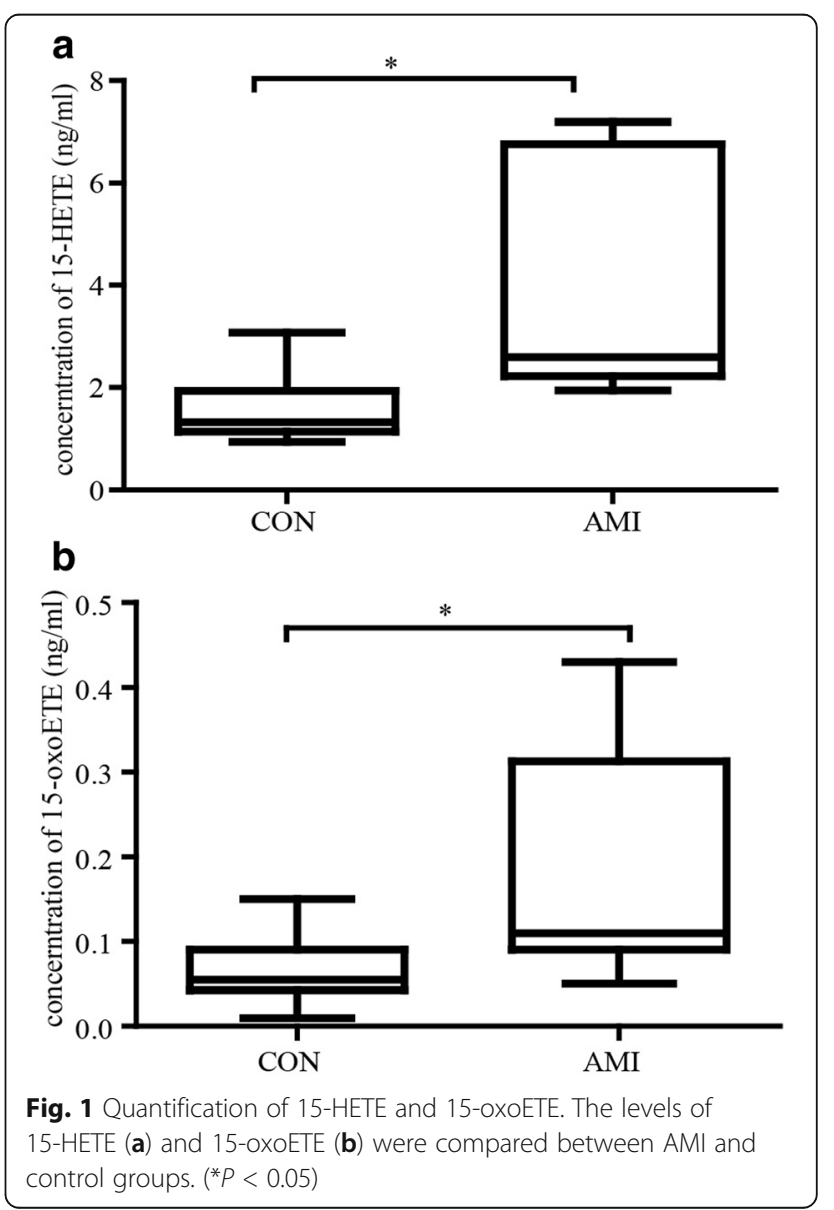




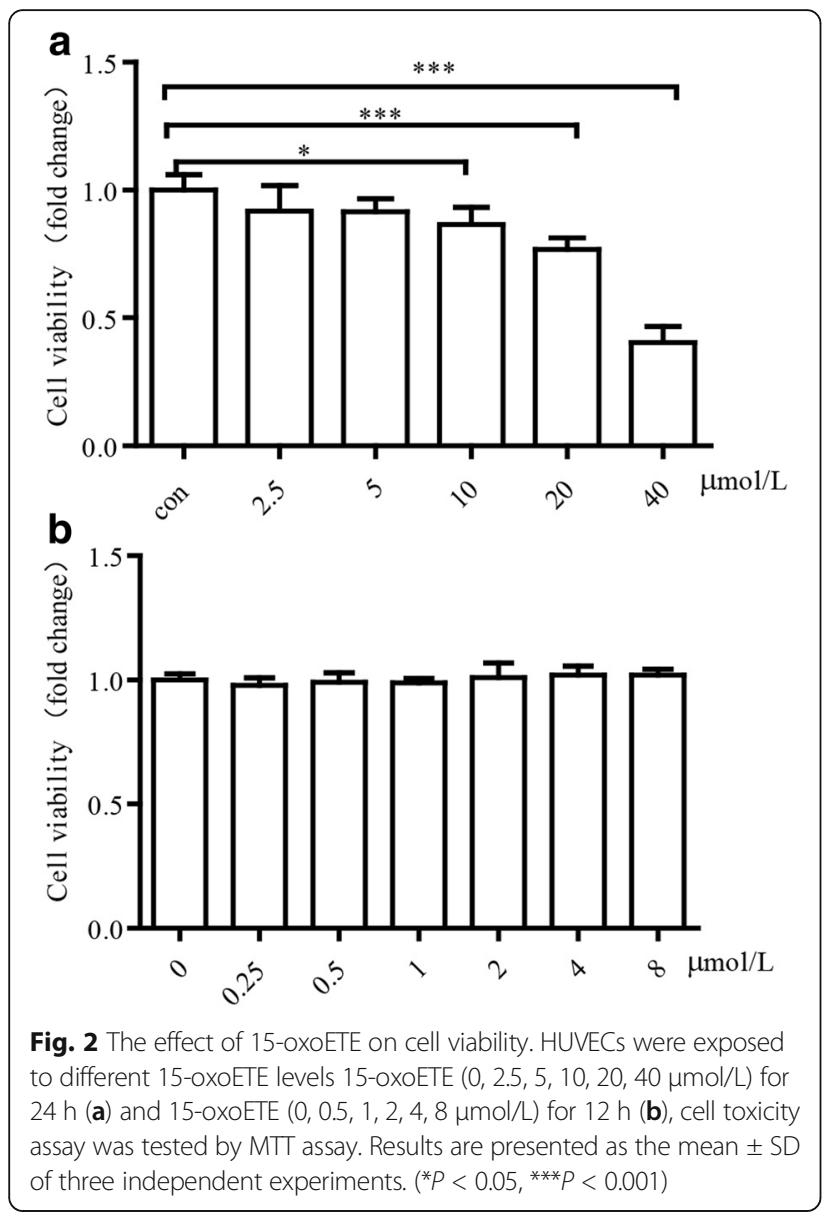

Adhesion of THP-1 cells to HUVECs was stimulated by 15-oxo-ETE in a dose-dependent manner as the amount of adherent THP-1 cells increased from $1.00 \pm 0.15$ to $1.28 \pm 0.26$ (Fig. 3a, b).

During the process of cell adhesion, cell adhesion molecules most likely play an important role in cell communication [25-27]. The cell ELISA results suggested that treatment of 15-oxo-ETE up-regulated E-selectin, but not ICAM-1 and VCAM-1 (Fig. 3c, d). When HUVECs were treated with $8 \mu \mathrm{mol} / \mathrm{L}$ of 15-oxo-ETE, expression of E-selectin reached $1.40 \pm 0.32$ fold increase compared with control (Fig. 3e). The protein level of E-selectin was also up-regulated as analyzed by Western blot (Fig. 3f).

15-oxo-ETE promotes THP-1 adhesion to mice aortic wall and increases E-selectin expression in mice aortic ECs

To further confirm the contribution of 15-oxo-ETE-induced monocyte adhesion ex vivo, we performed adhesion assays with aorta isolated from C57 mice and ApoE-/- mice. Treatment with 15-oxo-ETE make an increase in monocytes adhesion to the aorta of C57 mice, but no significant difference is showed (4.29 \pm 3.90 fold change, $P=0.1376$ ) (Fig. $4 \mathrm{a}, \mathrm{c}$ ) and E-selectin expression (2.89 \pm 1.93 fold change) (Fig. 4e). Coincidently, exposure of arteries from ApoE-/-mice to 15-oxo-ETE led to significantly increased monocyte adhesion (12.46 \pm 7.55 fold change, $P<0.05$ ) (Fig. $4 a, c)$ and $E-$ selectin expression (3.11 \pm 1.78 fold change) (Fig. 4e). However, no change was observed in the expression of ICAM-1and VCAM-1 compared with control (Fig. 4e).

\section{PKC activation is required for 15-oxoETE-induced monocyte adhesion}

It has been known that the activity of PKC plays an important role in regulating endothelial dysfunction, including inflammation and adhesion [23]. PKC activity was examined in HUVECs treated with 15-oxoETE. In the present study, 15-oxoETE accelerated PKC phosphorylation compared with control in a dose-dependent manner (Fig. 5a). Interestingly, staurosporine treatment significantly diminished the increased monocytes adhesion in every concentration level of 15-oxoETE, especially in higher concentrations of $2-8 \mu \mathrm{mol} / \mathrm{L}$ (Fig. 5b). These data indicated that the activation of $\mathrm{PKC}$ was responsible for 15-oxoETE-induced monocytes recruitment.

\section{Discussion}

15-oxoETE is firstly found in rabbit lung, which is produced from 15-PGDH-mediated oxidation of 15-HETE [28]. Cong Wei et al. found that 15-oxoETE inhibited endothelial proliferation in a dose-dependent manner [29]. There was also a study suggested that 15-oxoETE promoted carotid artery construction during hypoxia [30]. These results indicated that 15-oxoETE might play an important role in atherosclerosis. Conversely, 15-oxoETE is found to protect pulmonary arterial smooth muscle cells against serum deprivation-induced apoptosis, indicating a potential therapeutical role of 15-oxoETE in pulmonary arterial hypertension [31]. What's more, 15-oxoETE was reported to mediate pro-inflammatory signaling by activating anti-inflammatory Nrf2 signaling and downregulating pro-inflammatory cytokine [32]. Taken together, this interesting metabolite of AA remains a biological curiosity in atherosclerotic pathophysiology. Thus, this study attempts to demonstrate that 15-oxoETE promote pathological process of atherosclerosis by accelerating monocytes recruitment.

More recently, 15-oxo-ETE was identified as a metabolite of AA in primary human monocytes with IL-4 treatment. They indicated that 15-oxoETE had a short half-life of only $11 \mathrm{~min}$, and then crossed the cell membrane rapidly [29]. It may be the reason why people found 15-HETE but not 15-oxoETE is the main AA metabolite in atherosclerotic plaque. Interestingly, the present UPLC/MS/MS analysis showed that both 15HETE and 15-oxoETE level is elevated in AMI patients (Table 2, Fig. 1). Thus, we hypothesized 15-oxoETE was 


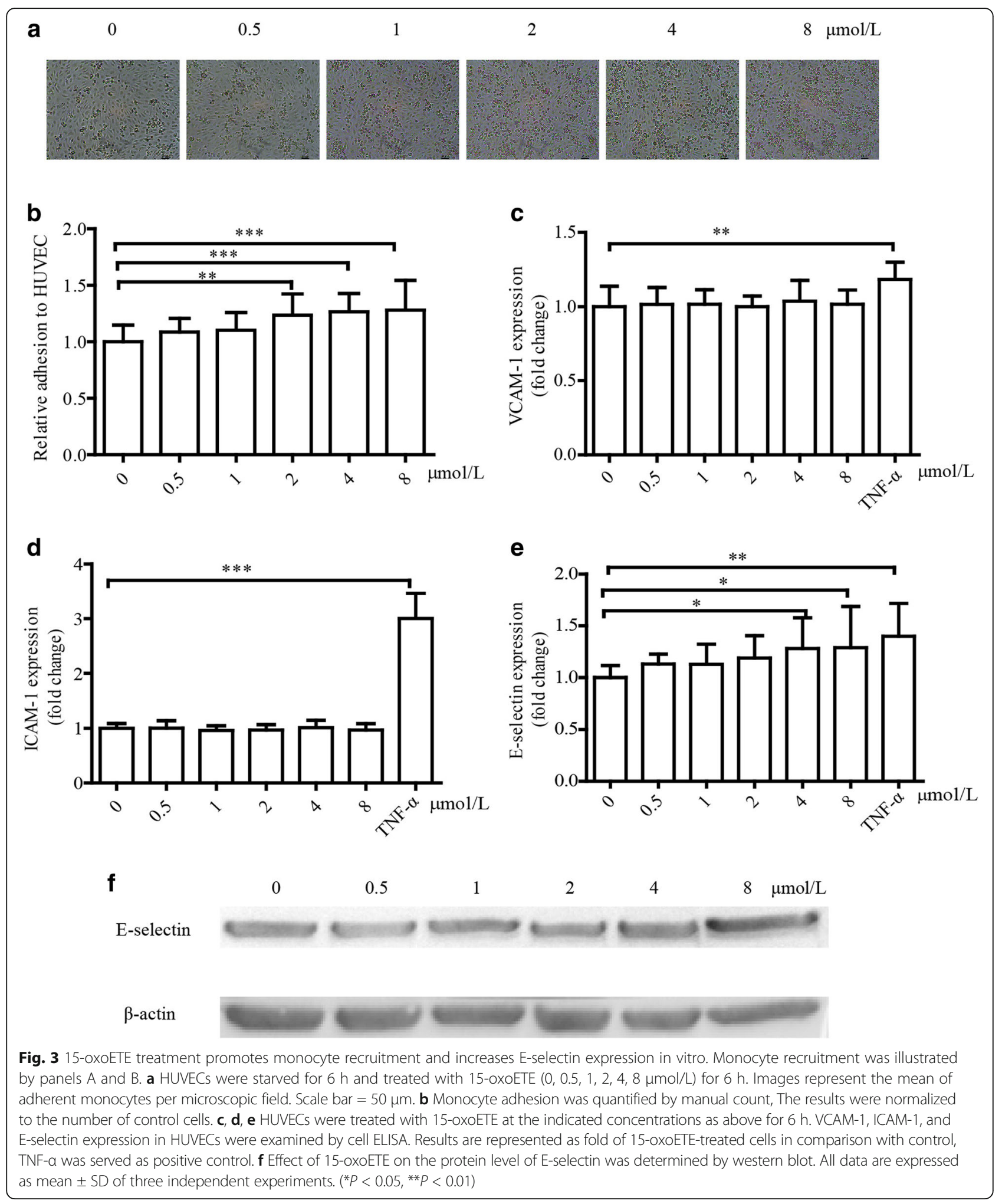

released from macrophagocytes in atherosclerotic plaque to affect vascular function.

Vascular inflammation and monocyte adhesion are critical events in the initiation of atherosclerosis $[25,33]$.
The increased expression of cellular adhesion molecules, such as VCAM-1 E-selectin and ICAM-1, plays an important role in endothelial inflammation and is essential to recruiting monocytes from the circulation [33, 34]. 


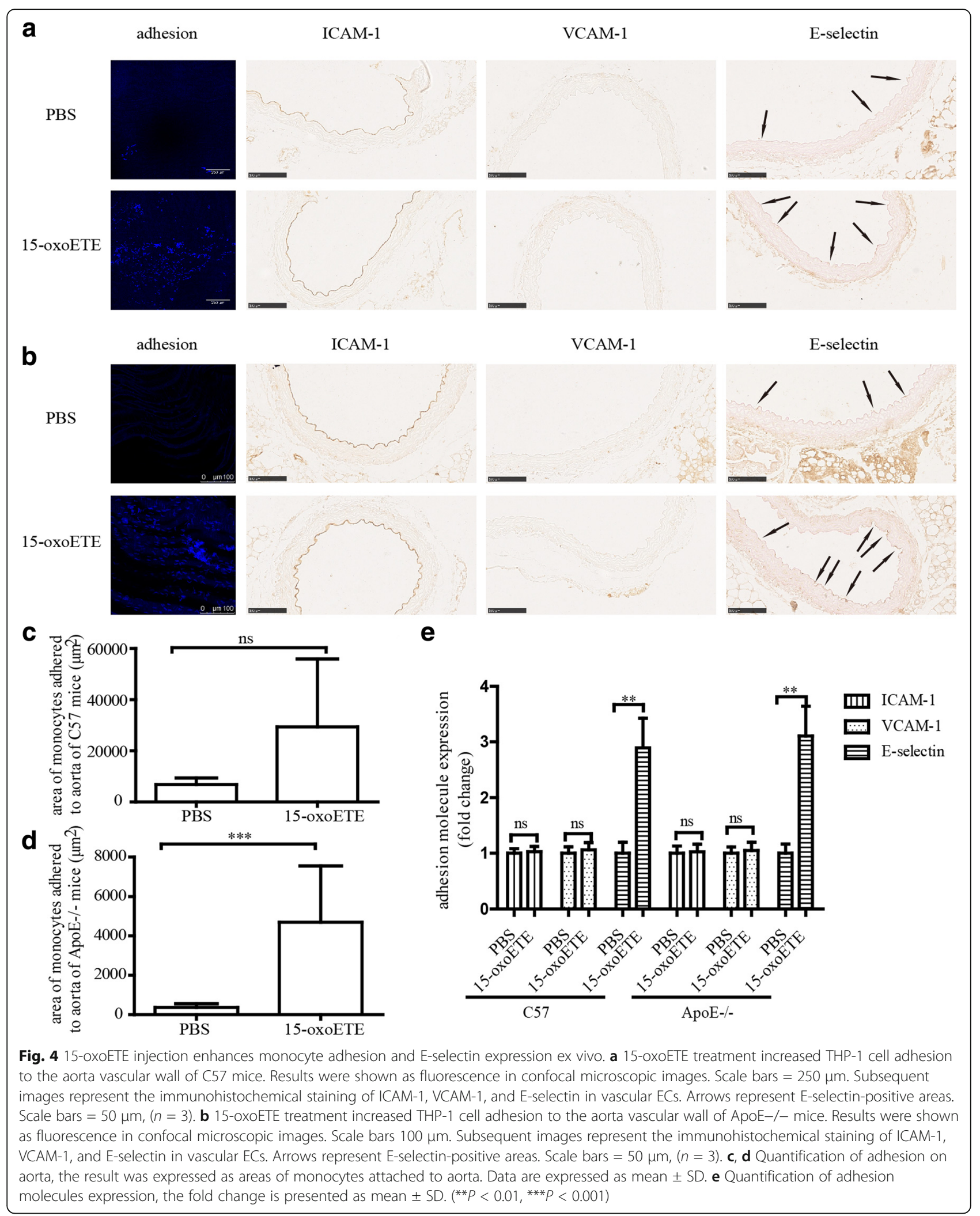




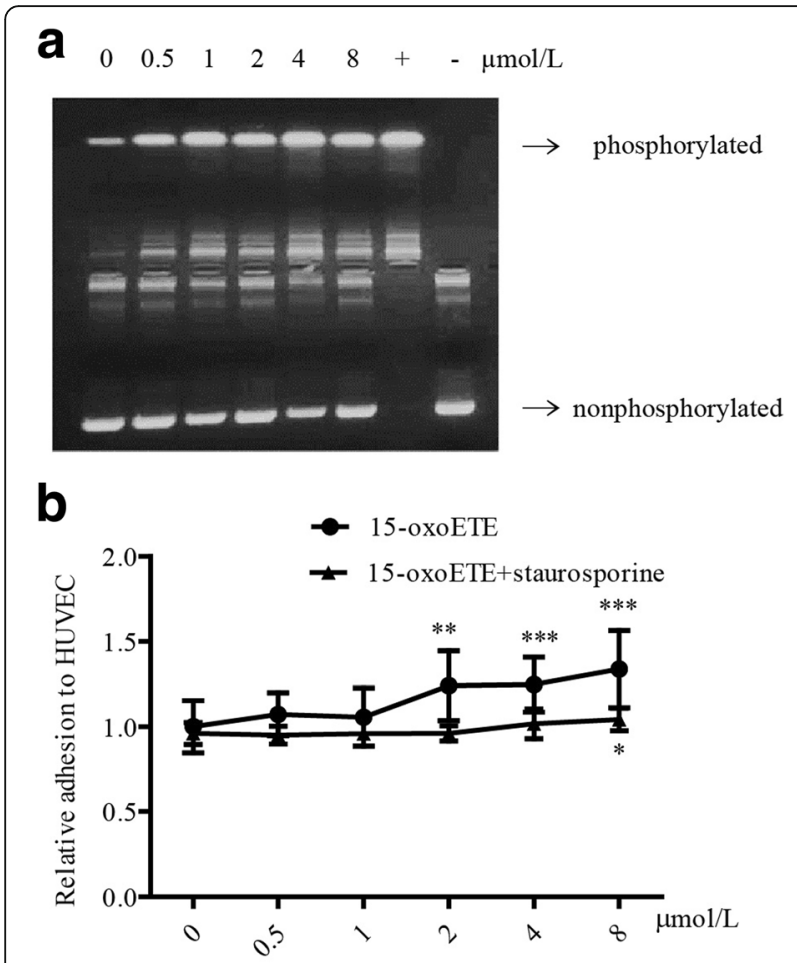

Fig. 5 15-OXO-ETE-induced monocyte adhesion is attributed to PKC activation. a HUVECs were treated with 15-OxoETE $(0,0.5,1,2,4$, $8 \mu \mathrm{mol} / \mathrm{L})$ for $6 \mathrm{~h}$ in serum-free media. Cell lysates from 15-oxoETEtreated cells were analyzed by the PepTag nonradioactive PKC assay. b Monocyte adhesion was measured in the presence or absence of staurosporine $(2.5 \mathrm{nmol} / \mathrm{L})$, the results are presented as the mean $\pm \mathrm{SD}$ of three independent experiments $\left({ }^{*} P<0.05,{ }^{*} P<0.01\right.$, ${ }^{* *} P<0.001$, compared with control)

The present study suggested that 15-oxoETE positively regulated E-selectin expression in HUVECs and increased monocyte adhesion to HUVECs (Fig. 3). Consistently, the result is further tested in both C57 and ApoE-/- mice. What's more, the adhesion change is more significant in ApoE-/- mice than C57 mice (Fig. 4). These data revealed that 15-oxoETE might be a risk factor of cardiovascular disease in both health groups and high-risk groups.

PKC activation strengthens the adhesion reaction of endothelial cells with monocytes [35, 36]. Four PKC isoforms have been identified in human ECs, namely $\mathrm{PKC}-\alpha, \mathrm{PKC}-\delta, \mathrm{PKC}-\varepsilon$, and $\mathrm{PKC}-\zeta$ [27]. Although the present study did not explore the roles of different PKC isoforms in 15-OXO-ETE-treated ECs, we showed that total PKCs activity was increased in 15-oxoETE-treated ECs (Fig. 5a). Moreover, increased monocyte adhesion was significantly diminished by PKC inhibitor (Fig. 5b). Therefore, we speculate that PKC activation may play an important role in 15-oxoETE induced monocyte adhesion.

\section{Conclusion}

These studies provide a novel role of 15-oxoETE in atherosclerosis. 15-oxoETE promotes E-selectin expression and PKC-dependent monocyte adhesion, which indicating that 15-oxoETE is probably a potential risk factor of atherosclerosis. This question will be better clarified until abundant convictive clinical data will be given.

\section{Additional file}

Additional file 1: The details of patients are provided as follow. (DOCX $32 \mathrm{~kb}$ )

\begin{abstract}
Abbreviations
15(S)-HETE: 15(S)-hydroperoxy-5,8,11,13-(Z,Z,Z,E)-eicosatetraenoic acid; 15-HpETEs: 15-hydroperoxyeicosatetraenoic acids; 15-oxo-ETE: 15-oxo5,8,11,13-(Z,Z,Z,E)-eicosatetraenoic acid; AA: Arachidonic acid; AMI: Acute myocardial infarction; HUVECs: Human umbilical vein endothelial cells; LAD: Left anterior descending; LC-MS/MS: Liquid chromatography and online tandem mass spectrometry; LCX: Left circumflex artery; LDL: Low-density lipoprotein; Non-STEMI: Non ST-segment elevated myocardial infarction; PGDH: 15-hydroxyprostaglandin dehydrogenase; PUFA: Polyunsaturated fatty acid; RCA: Right coronary artery; SAP: Stable angina pectoris; STEMI: ST-segment elevated myocardial infarction
\end{abstract}

\section{Acknowledgements}

We thank Miss Liang J Peking in University Health Science Center for providing valuable suggestion.

\section{Funding}

This work was supported by the National Natural Science Foundation of China $(81,450,012 ; 81,172,500 ; 91,639,108 ; 81,370,235)$.

\section{Availability of data and materials}

The datasets used and/or analysed during the current study available from the corresponding author on reasonable request.

\section{Authors' contributions}

BCh and LZh conceived and designed the experiments. CG contributed to sample and clinical data collection. GM and BP performed the experiments and analyzed the data. BCh, LZh and BP participated in data interpretation. GM wrote the manuscript. BCh supervised the experimental design, data analysis and manuscript preparation and final revision. SR contributed to the acquisition of the detail data of patient clinical characters and analyzed the data. YG and LW contributed to the analysis and interpretation of clinical data. All authors read and approved the final manuscript.

\section{Competing interests}

The authors declare that they have no competing interests.

\section{Consent for publication}

Not applicable.

\section{Ethics approval and consent to participate}

The study was designed and carried out in accordance with the principles of the declaration of Helsinki, and approved by the Ethics Committee of Beijing TianTan hospital. All patients provided written informed consent.

\section{Publisher's Note}

Springer Nature remains neutral with regard to jurisdictional claims in published maps and institutional affiliations.

\section{Author details}

'Department of Cardiology, Beijing Tian Tan Hospital, Capital Medical University, Beijing 100050, China. ${ }^{2}$ The Institute of Cardiovascular Sciences and Institute of Systems Biomedicine, School of Basic Medical Sciences, and Key Laboratory of Molecular Cardiovascular Sciences of Ministry of Education, 
Peking University Health Science Center, Beijing 100191, China. ${ }^{3}$ Tai Zhou Municipal Hospital, Taizhou 318000, China. ${ }^{4}$ Department of Cardiovascular Medicine, Fujian Provincial Hospital, Fuzhou, China. ${ }^{5}$ Department of Nephrology, Union Hospital, Fujian Medical University Union Hospital, Fuzhou, China.

Received: 28 January 2017 Accepted: 15 June 2017

Published online: 12 July 2017

\section{References}

1. Powell WS, Rokach J. Biosynthesis, biological effects, and receptors of hydroxyeicosatetraenoic acids (HETEs) and oxoeicosatetraenoic acids (oxo-ETEs) derived from arachidonic acid. Biochim Biophys Acta. 2015;1851:340

2. Haeggstrom JZ, Funk CD. Lipoxygenase and leukotriene pathways: biochemistry, biology, and roles in disease. Chem Rev. 2011;111:5866.

3. Brash AR, Boeglin WE, Chang MS. Discovery of a second 15S-lipoxygenase in humans. Proc Natl Acad Sci U S A. 1997;94:6148.

4. Wittwer J, Hersberger M. The two faces of the 15-lipoxygenase in atherosclerosis. Prostaglandins Leukot Essent Fatty Acids. 2007;77:67.

5. Gertow K, et al. 12- and 15-lipoxygenases in human carotid atherosclerotic lesions: associations with cerebrovascular symptoms. Atherosclerosis. 2011;215:411.

6. Hulten LM, et al. 15-Lipoxygenase-2 is expressed in macrophages in human carotid plaques and regulated by hypoxia-inducible factor-1alpha. Eur J Clin Investig. 2010:40:11.

7. Hiltunen T, Luoma J, Nikkari T, Yla-Herttuala S. Induction of 15-lipoxygenase mRNA and protein in early atherosclerotic lesions. Circulation. 1995:92:3297.

8. Sukhanov S, et al. Insulin-like growth factor I reduces lipid oxidation and foam cell formation via downregulation of 12/15-lipoxygenase. Atherosclerosis. 2015;238:313

9. Takeda S, et al. Cannabidiol-2',6'-dimethyl ether as an effective protector of 15-lipoxygenase-mediated low-density lipoprotein oxidation in vitro. Biol Pharm Bull. 2011;34:1252

10. Rankin SM, Parthasarathy S, Steinberg D. Evidence for a dominant role of lipoxygenase(s) in the oxidation of LDL by mouse peritoneal macrophages. J Lipid Res. 1991;32:449.

11. Rydberg EK, et al. Hypoxia increases LDL oxidation and expression of 15-lipoxygenase-2 in human macrophages. Arterioscler Thromb Vasc Biol. 2004;24:2040.

12. Kundumani-Sridharan V, Dyukova E, Hansen DR, Rao GN. 12/15-Lipoxygenase mediates high-fat diet-induced endothelial tight junction disruption and monocyte transmigration: a new role for 15(S)-hydroxyeicosatetraenoic acid in endothelial cell dysfunction. J Biol Chem. 2013;288:15830.

13. Waddington E, Sienuarine K, Puddey I, Croft K. Identification and quantitation of unique fatty acid oxidation products in human atherosclerotic plaque using high-performance liquid chromatography. Anal Biochem. 2001:292:234.

14. Singh NK, et al. 15-Lipoxygenase-1-enhanced Src-Janus kinase 2-signal transducer and activator of transcription 3 stimulation and monocyte chemoattractant protein-1 expression require redox-sensitive activation of epidermal growth factor receptor in vascular wall remodeling. J Biol Chem. 2011:286:22478

15. Wang S, et al. Activation of ERK pathway is required for 15-HETE-induced angiogenesis in human umbilical vascular endothelial cells. J Recept Signal Transduct Res. 2016;36:225

16. Kotla S, Singh NK, Traylor JJ, Orr AW, Rao GN. ROS-dependent Syk and Pyk2-mediated STAT1 activation is required for 15(S)hydroxyeicosatetraenoic acid-induced CD36 expression and foam cell formation. Free Radic Biol Med. 2014;76:147.

17. Matsuzawa Y, Guddeti RR, Kwon TG, Lerman LO, Lerman A. Treating coronary disease and the impact of endothelial dysfunction. Prog Cardiovasc Dis. 2015;57:431.

18. Bonetti PO, Lerman LO, Lerman A. Endothelial dysfunction: a marker of atherosclerotic risk. Arterioscler Thromb Vasc Biol. 2003:23:168

19. Park KH, Park WJ. Endothelial Dysfunction: Clinical Implications in Cardiovascular Disease and Therapeutic Approaches. J Korean Med Sci. 2015;30:1213.

20. Santos-Gallego CG, Picatoste B, Badimon JJ. Pathophysiology of acute coronary syndrome. Curr Atheroscler Rep. 2014;16:401.
21. Jaffe EA, Nachman RL, Becker CG, Minick CR. Culture of human endothelial cells derived from umbilical veins. Identification by morphologic and immunologic criteria. J Clin Invest. 1973;52:2745.

22. Pan B, et al. Hypochlorite-induced oxidative stress elevates the capability of HDL in promoting breast cancer metastasis. J Transl Med. 2012;10:65.

23. Durpes $M C$, et al. PKC-beta activation inhibits IL-18-binding protein causing endothelial dysfunction and diabetic atherosclerosis. Cardiovasc Res. 2015;106:303.

24. Pan B, et al. Diabetic HDL is dysfunctional in stimulating endothelial cell migration and proliferation due to down regulation of SR-BI expression. PLoS One. 2012:7:e48530.

25. Galkina E, Ley K. Vascular adhesion molecules in atherosclerosis. Arterioscler Thromb Vasc Biol. 2007:27:2292.

26. Zhao $Y$, et al. Deficiency of the NR4A orphan nuclear receptor NOR1 decreases monocyte adhesion and atherosclerosis. Circ Res. 2010;107:501.

27. Min JK, et al. TNF-related activation-induced cytokine enhances leukocyte adhesiveness: induction of ICAM-1 and VCAM-1 via TNF receptor-associated factor and protein kinase C-dependent NF-kappaB activation in endothelial cells. J Immunol. 2005;175:531.

28. Bergholte JM, Soberman RJ, Hayes R, Murphy RC, Okita RT. Oxidation of 15-hydroxyeicosatetraenoic acid and other hydroxy fatty acids by lung prostaglandin dehydrogenase. Arch Biochem Biophys. 1987:257:444.

29. Wei C, Zhu P, Shah SJ, Blair IA. 15-oxo-Eicosatetraenoic acid, a metabolite of macrophage 15-hydroxyprostaglandin dehydrogenase that inhibits endothelial cell proliferation. Mol Pharmacol. 2009;76:516.

30. Wang D, Liu Y, Lu P, Zhu D, Zhu Y. 15-oxo-ETE-induced internal carotid artery constriction in hypoxic rats is mediated by potassium channels. Physiol Res. 2015;65:391.

31. Sugumaran PK, et al. 15-oxo-Eicosatetraenoic acid prevents serum deprivation-induced apoptosis of pulmonary arterial smooth muscle cells by activating pro-survival pathway. Prostaglandins Leukot Essent Fatty Acids. 2014;90:89.

32. Snyder NW, et al. 15-Oxoeicosatetraenoic acid is a 15-hydroxyprostaglandin dehydrogenase-derived electrophilic mediator of inflammatory signaling pathways. Chem Biol Interact. 2015;234:144.

33. Herbin $\mathrm{O}$, et al. Monocyte Adhesion and Plaque Recruitment During Atherosclerosis Development Is Regulated by the Adapter Protein Chat-H/SHEP1. Arterioscler Thromb Vasc Biol. 2016;36:1791.

34. Pankow JS, et al. Circulating cellular adhesion molecules and risk of diabetes: the Multi-Ethnic Study of Atherosclerosis (MESA). Diabet Med. 2016;33:985

35. Li L, et al. Mechanism of PKC activity affecting the adhesion reaction of endothelial cells with monocytes. Int J Cardiol. 2015;182:361.

36. Geraldes P, King GL. Activation of protein kinase $C$ isoforms and its impact on diabetic complications. Circ Res. 2010;106:1319.

\section{Submit your next manuscript to BioMed Central and we will help you at every step:}

- We accept pre-submission inquiries

- Our selector tool helps you to find the most relevant journal

- We provide round the clock customer support

- Convenient online submission

- Thorough peer review

- Inclusion in PubMed and all major indexing services

- Maximum visibility for your research

Submit your manuscript at www.biomedcentral.com/submit 